State-of-the-art density matrix renormalization group and coupled cluster theory studies of the nitrogen binding curve

Garnet Kin-Lic ChanMihály Kállay and Jürgen Gauss

Citation: 121, (2004); doi: 10.1063/1.1783212

View online: http://dx.doi.org/10.1063/1.1783212

View Table of Contents: http://aip.scitation.org/toc/jcp/121/13

Published by the American Institute of Physics 


\title{
ARTICLES
}

\section{State-of-the-art density matrix renormalization group and coupled cluster theory studies of the nitrogen binding curve}

\author{
Garnet Kin-Lic Chan ${ }^{a)}$ \\ Department of Chemistry, University of Cambridge, Cambridge CB2 1EW, United Kingdom \\ Mihály Kállay and Jürgen Gauss \\ Institut für Physikalische Chemie, Universität Mainz, D-55099 Mainz, Germany
}

(Received 27 May 2004; accepted 24 June 2004)

\begin{abstract}
We study the nitrogen binding curve with the density matrix renormalization group (DMRG) and single-reference and multireference coupled cluster (CC) theory. Our DMRG calculations use up to 4000 states and our single-reference CC calculations include up to full connected hextuple excitations. Using the DMRG, we compute an all-electron benchmark nitrogen binding curve, at the polarized, valence double-zeta level (28 basis functions), with an estimated accuracy of $0.03 \mathrm{~m} E_{h}$. We also assess the performance of more approximate DMRG and CC theories across the nitrogen curve. We provide an analysis of the relative strengths and merits of the DMRG and CC theory under different correlation conditions. (C) 2004 American Institute of Physics.
\end{abstract}

[DOI: $10.1063 / 1.1783212]$

\section{INTRODUCTION}

High-accuracy numerical studies of the Schrödinger equation allow us to assess approximate methods and the contribution of higher-level corrections to molecular properties. These studies are of particular interest in challenging systems, which possess complicated electronic structure.

In this work, we examine the well-known problem of the nitrogen binding curve. As the nitrogen triple bond is stretched, the electronic structure changes from singlereference to highly multireference, and consequently a balanced description across the potential energy curve has traditionally been difficult to achieve. ${ }^{1,2,3,4-6}$

Here, we use the density matrix renormalization group (DMRG) and high-level coupled cluster (CC) theories to compute a benchmark, all-electron, nitrogen binding curve within the Dunning $c c-p$ VDZ basis. ${ }^{7}$ These calculations thus go beyond previous full configuration interaction (FCI) studies, which used the frozen-core $(10 e)$ approximation. ${ }^{1,6}$ Allelectron FCI calculations [which retain all elements of the configuration interaction $(\mathrm{CI})$ vector] for this system remain currently out of reach (the $D_{2 h}$ space contains roughly 1.8 $\times 10^{11}$ determinants). While the DMRG and CC theories should be seen as providing a hierarchy of approximations to the FCI result, our calculations have been carried out to a very high level $(M=4000$ for DMRG, and up to hextuple excitations for CC theory), yielding near-FCI quality numerical results. Our best DMRG calculations yield an estimated residual error across the nitrogen binding curve of better than $0.03 \mathrm{M} E_{h}$, and, in the equilibrium region, even higher accuracy is achieved by our best $\mathrm{CC}$ results.

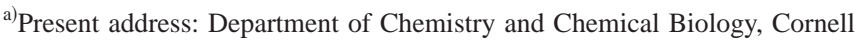
University, Ithaca, NY 14853-1301.
}

In addition to the benchmark calculations, we examine the behavior of both approximate DMRG and CC (single reference and multireference) theories for the nitrogen dissociation problem. A particular question we seek to answer is the relative suitability of the DMRG and CC theories in bond-breaking situations. This study leads us to conclude with an analysis of the relative merits of the two approaches under different correlation conditions.

\section{METHODOLOGY}

\section{A. DMRG}

The DMRG is a method to systematically approximate the solution of the Schrödinger equation. It has been widely applied to study strongly-correlated quantum lattices ${ }^{8,9}$ and has more recently been developed as a quantum chemical method. ${ }^{10-15}$ As a detailed description of our algorithm and implementation may be found in Refs. 12 and 14 we shall concentrate on the conceptual aspects of the method.

We first partition orbitals into two groups: a left and right block with $l$ and $r$ orbitals. In general, the Fock space of each block $F_{l}, F_{r}$ is spanned by a set of many-particle functions $\left\{\chi_{l}\right\},\left\{\chi_{r}\right\}$, and thus the exact wave function of the system may be written as

$$
\Psi=\sum_{\lambda \mu} d_{\lambda \mu}\left|\chi_{l}^{\lambda}\right\rangle\left|\chi_{r}^{\mu}\right\rangle=\sum_{\lambda} c_{\lambda}\left|\chi_{l}^{\lambda}\right\rangle\left|\phi_{r}^{\lambda}\right\rangle,
$$

where $\phi_{r}^{\lambda}=\Sigma_{\mu} d_{\lambda \mu} \chi_{r}^{\mu}$, and the sum in Eq. (1) is subject to necessary constraints, e.g., if the system has a definite number of particles $N$, then the number of particles in $\chi_{l}$ and $\phi_{r}$ must add up to $N$.

In the DMRG, we seek the best approximation of rank $4 M$ to $\Psi$ Ref. 16 , and thus the DMRG Ansatz is 


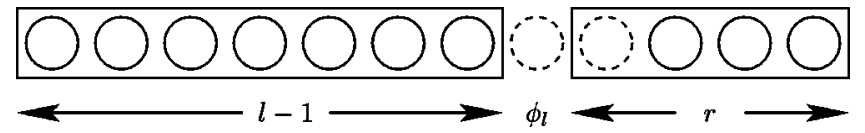

FIG. 1. A typical block configuration, with $l-1$ orbitals (circles) in the left block, $r$ orbitals in the right block, and a single orbital (dashed circle) $\phi_{l}(4$ states) to be added to the left block. For simplicity, the orbital that is added to the right block (dashed circle) is considered as part of the right block here.

$$
\Psi=\sum_{\lambda}^{4 M} c_{\lambda}\left|\psi_{l}^{\lambda}\right\rangle\left|\psi_{r}^{\lambda}\right\rangle
$$

where $\psi_{l}^{\lambda}, \psi_{r}^{\lambda}$ are now optimal many-particle functions in the Fock spaces of the left and right blocks. The Ansatz (2) amounts to a singular value decomposition of $\Psi$ where $\psi_{l}^{\lambda}, \psi_{r}^{\lambda}$ are the singular vectors and $c_{\lambda}$ are the weights. Equivalently, in a procedure from which the method derives its name, we can compute $\psi_{l}^{\lambda}$ as the eigenvectors of the reduced density matrix $\Gamma=\operatorname{Tr}_{\{r\}}|\Psi\rangle\langle\Psi|$, where we have traced over the states in the right-hand block. Because the functions $\psi_{l}^{\lambda}, \psi_{r}^{\lambda}$ are optimal many-particle functions, and not simply determinants, it is possible for Eq. (2) to provide a compact representation of $\Psi$ for relatively small values of $M$.

The objective of the DMRG algorithm is then to determine the optimal functions $\psi_{l}^{\lambda}, \psi_{r}^{\lambda}$. To facilitate this, the DMRG assumes an additional nested structure-typical of a renormalization group theory-that relates the functions in successively larger blocks,

$$
\left\{\psi_{l}^{\lambda}\right\} \in\left\{\phi_{l}\right\} \otimes\left\{\psi_{l-1}^{\lambda}\right\},
$$

that is, the optimal functions for the block $l$ are contained within the product Fock space of the smaller block $l-1$ and orbital $\phi_{l}$ (see Fig. 1). At each length of block $l$, the optimal $M$ functions $\psi_{l}^{\lambda}$ are determined by solving the Schrödinger equation with the best current representations $\left\{\psi_{r}^{\lambda}\right\}$ and $\left\{\psi_{l-1}^{\lambda}\right\}$ in the tensor product space of $O\left(16 M^{2}\right)$ functions, and constructing the reduced density matrix of the left-hand block to determine the $O(4 M)$ eigenvectors $\psi_{l}^{\lambda}$ (of which we retain $M$ ). Thus DMRG calculations are performed in an iterative set of sweeps, where in each sweep, one block is increased in size at the expense of the other, while the representations of the spaces $\left(\left\{\psi_{l}^{\lambda}\right\}\right.$ or $\left\{\psi_{r}^{\lambda}\right\}$ depending on the direction of the sweep) are improved.

As we sweep through the orbitals to construct our spaces in Eq. (3), we need to specify an order in which the orbitals are traversed. In one-dimensional systems, a natural ordering consistent with the hierarchical space structure (3) exists and in such systems, the DMRG is particularly powerful. However, we do not have this natural ordering in most molecules, and in practice, we commonly order by grouping orbitals together to minimize long-range correlations (and thus the number of interactions between the left and right blocks), or simply energy order by orbital eigenvalue. ${ }^{12,17,18}$

The DMRG wave function exists within a product Ansatz, and thus with a physical ordering, the DMRG is a sizeconsistent theory. Consider a system with two noninteracting widely-separated fragments $A, B$ and with orbitals $\{a\}$ on $A$ and $\{b\}$ on B. Now order the orbitals, $\{a\}\{b\}$, so that the orbitals on $A$ and $B$ form two disjoint sets. If we now place the dividing line between the left and right blocks between $\{a\}$ and $\{b\}$, then the DMRG $\Psi$ is immediately in the separable size-consistent product form $\Psi=\psi_{A} \psi_{B},{ }^{19}$ where $\psi_{A}, \psi_{B}$ are the corresponding rank $M$ DMRG wave functions for $A, B$.

\section{B. Coupled cluster theory}

Excellent treatments of CC theory may be found in the literature ${ }^{20,21}$ and we provide only a brief review. In CC theory, the wave function is expressed by the exponential Ansatz

$$
\Psi=e^{T}|0\rangle,
$$

where $|0\rangle$ is the reference, usually a Hartree-Fock determinant, and $T$ is an excitation operator,

$$
T=\sum_{n} T_{n}
$$

e.g.,

$$
\begin{aligned}
& T_{1}=\sum_{i a} t_{i}^{a} a_{a}^{\dagger} a_{i}, \\
& T_{2}=\frac{1}{4} \sum_{i j a b} t_{i j}^{a b} a_{a}^{\dagger} a_{b}^{\dagger} a_{i} a_{j},
\end{aligned}
$$

where $i, j$ denote orbitals occupied in $|0\rangle$ and $a, b$ denote the corresponding unoccupied virtuals. The important feature of the CC Ansatz is the separation of correlation into connected and disconnected components; for example, double excitations from $i j \rightarrow a b$ are expressed as the sum of a (disconnected) product of single excitations due to $(1 / 2) T_{1}^{2}$ and a connected contribution $T_{2}$. A truncated $T$ operator yields a good representation of the wave function when the neglected connected components are small. In addition, truncated CC Ansätze are size consistent since $e^{T_{A B}}=e^{T_{A}+T_{B}}=e^{T_{A}} e^{T_{B}}$, if $T_{A}$ and $T_{B}$ act only on $A$ and $B$, respectively, since the CC equations are fully connected.

The CC equations to determine the $T$ amplitudes are nonlinear equations with a number of terms that grow factorially with excitation order $n$. This has led to several different computational strategies to handle the complexity of high order CC theory. ${ }^{22-26}$ In this work, we use a string-based algorithm $^{23,27}$ to evaluate excitations of arbitrary order.

\section{Multireference coupled cluster and configuration interaction}

In our study of approximate methods (Sec. IIIC) we present calculations using the multireference configuration interaction (MRCI) and multireference coupled cluster (MRCC) theories. These were developed to describe the kind of correlation problems we encounter in the current work, i.e., bond breaking, where the Hartree-Fock reference is a poor representation. In these methods, the Hartree-Fock reference is replaced by a set of reference determinants, often chosen to be a complete active space, and usually single and double excitations out of the reference determinants are con- 
TABLE I. Frozen-core correlation energies (relative to the RHF reference) for DMRG, CC, FCI, and RHF total energies. All results in $E_{h}$. Italics denote unconverged digits.

\begin{tabular}{|c|c|c|c|c|c|c|}
\hline & $2.118 a_{0}$ & $2.4 a_{0}$ & $2.7 a_{0}$ & $3.0 a_{0}$ & $3.6 a_{0}$ & $4.2 a_{0}$ \\
\hline RHF & -108.949378 & -108.866811 & -108.737400 & -108.606226 & -108.384757 & -108.222899 \\
\hline FCI & -0.328961 & -0.371586 & -0.422905 & -0.479983 & -0.610149 & -0.744051 \\
\hline DMRG: 1000 & -0.3286 & -0.3712 & -0.4225 & -0.4794 & -0.6095 & -0.7434 \\
\hline DMRG: 2000 & -0.32890 & -0.37151 & -0.42283 & -0.47986 & -0.61001 & a \\
\hline DMRG: 4000 & -0.328951 & -0.371575 & -0.422893 & -0.479963 & -0.610129 & $\mathrm{a}$ \\
\hline CCSD & -0.314493 & -0.350105 & -0.391098 & -0.434795 & -0.537926 & -0.703353 \\
\hline $\operatorname{CCSD}(\mathrm{T})$ & -0.327095 & -0.368499 & -0.417723 & -0.472283 & -0.616179 & -0.909065 \\
\hline CCSDT & -0.327122 & -0.367955 & -0.416071 & -0.469673 & -0.626097 & -0.857182 \\
\hline CCSDTQ & -0.328732 & $-0.371003^{b}$ & $-0.421548^{\mathrm{b}}$ & $-0.47757^{\mathrm{b}}$ & $-0.6096^{\mathrm{b}}$ & $\mathrm{c}$ \\
\hline CCSDTQP & -0.328940 & a & a & a & c & $\mathrm{c}$ \\
\hline CCSDTQPH & -0.328959 & a & a & a & $\mathrm{c}$ & $\mathrm{c}$ \\
\hline MRCI & -0.322307 & -0.364763 & -0.415928 & -0.472865 & -0.602969 & -0.737138 \\
\hline MRCC & -0.327446 & -0.370058 & -0.421287 & -0.478194 & -0.607962 & -0.741805 \\
\hline UHF-CCSD & -0.314493 & -0.349551 & -0.390925 & -0.443632 & -0.588181 & -0.734422 \\
\hline UHF-CCSD(T) & -0.327095 & -0.365210 & -0.409908 & -0.459480 & -0.595061 & -0.737125 \\
\hline UHF-CCSDT & -0.327122 & -0.367663 & -0.415941 & -0.469972 & -0.602663 & -0.740928 \\
\hline UHF-CCSDTQ & -0.328732 & -0.370936 & -0.421467 & -0.476969 & -0.605808 & -0.742138 \\
\hline UHF-CCSDTQP & -0.328940 & -0.371445 & -0.422557 & -0.479164 & -0.607796 & -0.742907 \\
\hline UHF-CCSDTQPH & -0.328959 & -0.371514 & -0.422771 & -0.479781 & -0.609405 & -0.743224 \\
\hline
\end{tabular}

${ }^{a}$ Not computed due to computer time constraints.

${ }^{\mathrm{b}}$ Taken from Ref. 1.

${ }^{\mathrm{c}}$ Not computed due to convergence difficulties.

sidered. In a MRCI calculation the Hamiltonian is simply diagonalized in this space. The main formal disadvantage of MRCI is the lack of size consistency.

Among the several multireference generalizations of CC theory, we employ the state-selective MRCC Ansatz proposed originally by Oliphant and Adamowicz. ${ }^{28}$ In this approach a formal Fermi vacuum is chosen from the reference space and excitations out of the other reference functions are regarded as higher excitations from this determinant. The appealing feature of this method is that it retains the simplicity and the size-extensive nature of the single-reference CC formalism. More detailed descriptions of MRCI and MRCC theories may be found in Ref. 29.

\section{THE NITROGEN BINDING CURVE}

\section{A. Methodology}

Calculations on the nitrogen molecule were carried out using the Dunning $c c-p$ VDZ basis ${ }^{7}$ with spherical $d$ functions (28 basis functions in total). Six geometries were studied: $2.118\left(r_{e}\right), 2.4,2.7,3.0,3.6,4.2 a_{0}$. Frozen-core calculations employed both canonical restricted Hartree-Fock (RHF) and unrestricted Hartree-Fock (UHF) orbitals (energies given in Tables I and III), freezing the $1 \sigma_{g}, 1 \sigma_{u}^{*}$, or nitrogen 1s orbitals. All-electron calculations used canonical UHF orbitals. Molecular orbital calculations were performed and integrals were computed using MOLPRO. ${ }^{30}$

FCI calculations in $D_{2 h}$ symmetry were computed at the frozen-core level using the program of Kállay et al. ${ }^{23}$

DMRG calculations used the BLOCK program. ${ }^{14}$ Two warm-up sweeps at $M=600,800$ were carried out, followed by sweeps at $M=1000,2000,4000$. The number of sweeps at each $M$ value ranged from 6-17 sweeps at the $M=1000$ level, to 4-6 sweeps at the $M=4000$ level; all sweep energies are reported to the number of converged digits. Orbitals were ordered to minimize long-range interactions as measured by the one-electron integrals (orderings available as supplementary material). Since the one-electron integrals vanish between orbitals of different symmetry, these reorderings group orbitals of the same symmetry together. For technical reasons ${ }^{31}$ the DMRG calculations were carried out using only $C_{s}$ symmetry.

Single-reference CC calculations including up to hextuple excitations were carried out using the program of Kállay et $a l .{ }^{23} \mathrm{CCSD}(\mathrm{T})$ energies were obtained using ACES II. ${ }^{32}$ At the all-electron level we neglected hextuple excitations out of the nitrogen $1 s$ orbitals: this frozen-core approximation is denoted $\mathrm{H}-f c$. We estimate the error with respect to full CCSDTQPH since this approximation is in the range of $0.1-0.2 \mu E_{h}$. All CC calculations used the full symmetry group of the orbitals $\left(C_{2 v}\right.$ or $\left.D_{2 h}\right)$ and were converged to the $\mu E_{h}$ level.

MRCISD and MRCCSD calculations were obtained using the program of Kállay et $a .^{27} \mathrm{~A}$ complete active space (CAS) containing the nitrogen $3 \sigma_{g}^{+}, 1 \pi_{u}, 1 \pi_{g}, 3 \sigma_{u}^{+}$orbitals was used. The $(6,6) \mathrm{CAS}$ wave function was used as the reference space in the subsequent MRCCSD calculations. Multiconfigurational self-consistent-field calculations were performed and integrals were obtained using the COLUMBUS $^{33}$ suite of programs.

\section{B. Frozen-core calibration calculations}

Before presenting our all-electron results, we first present frozen-core calculations, which allow us to calibrate 
TABLE II. Errors in frozen-core correlation energies, relative to FCI (all energies in $\mathrm{m} E_{h}$ ). NPE $=\mathrm{abs}$ (maximum error - minimum error). Italics denote unconverged digits.

\begin{tabular}{lccccccc}
\hline \hline & $2.118 a_{0}$ & $2.4 a_{0}$ & $2.7 a_{0}$ & $3.0 a_{0}$ & $3.6 a_{0}$ & $4.2 a_{0}$ & NPE \\
\hline DMRG: 1000 & 0.3 & 0.4 & 0.4 & 0.6 & 0.7 & 0.6 & 0.4 \\
DMRG: 2000 & 0.06 & 0.07 & 0.08 & 0.12 & 0.14 & $\mathrm{a}$ & 0.08 \\
DMRG: 4000 & 0.010 & 0.011 & 0.012 & 0.019 & 0.020 & $\mathrm{a}$ & 0.010 \\
CCSD & 14.469 & 21.481 & 31.807 & 45.188 & 72.223 & 40.698 & 57.754 \\
CCSD(T) & 1.866 & 3.087 & 5.182 & 7.700 & -6.030 & -165.014 & 172.714 \\
CCSDT & 1.839 & 3.631 & 6.834 & 10.310 & -15.948 & -113.131 & 123.441 \\
CCSDTQ & 0.229 & $0.583^{\mathrm{b}}$ & $1.357^{\mathrm{b}}$ & $2.41^{\mathrm{b}}$ & $0.5^{\mathrm{b}}$ & $\mathrm{c}$ & 2.181 \\
CCSDTQP & 0.021 & $\mathrm{a}$ & $\mathrm{a}$ & $\mathrm{a}$ & $\mathrm{c}$ & $\mathrm{c}$ & $\mathrm{c}$ \\
CCSDTQPH & 0.002 & $\mathrm{a}$ & $\mathrm{a}$ & $\mathrm{a}$ & $\mathrm{c}$ & $\mathrm{c}$ & $\mathrm{c}$ \\
& & & & & & & \\
MRCI & 6.654 & 6.823 & 6.977 & 7.118 & 7.180 & 6.913 & 0.464 \\
MRCC & 1.515 & 1.529 & 1.618 & 1.789 & 2.187 & 2.247 & 0.732 \\
UHF-CCSD & 14.468 & 22.035 & 31.981 & 36.351 & 21.968 & 9.630 & 26.721 \\
UHF-CCSD(T) & 1.866 & 6.376 & 12.997 & 20.503 & 15.088 & 6.926 & 18.637 \\
UHF-CCSDT & 1.839 & 3.923 & 6.964 & 10.011 & 7.486 & 3.123 & 8.172 \\
UHF-CCSDTQ & 0.229 & 0.651 & 1.438 & 3.014 & 4.341 & 1.913 & 4.112 \\
UHF-CCSDTQP & 0.021 & 0.141 & 0.348 & 0.819 & 2.353 & 1.144 & 2.332 \\
UHF-CCSDTQPH & 0.002 & 0.073 & 0.134 & 0.202 & 0.744 & 0.827 & 0.825 \\
\hline \hline
\end{tabular}

${ }^{a}$ Not computed due to computer time constraints.

${ }^{\mathrm{b}}$ Taken from Ref. 1.

${ }^{\mathrm{c}}$ Not computed due to convergence difficulties.

our DMRG and CC methods against FCI. Our computed energies and errors from FCI are presented in Tables I, II, and Fig. 2.

We remark first on the convergence of the DMRG sweeps for fixed $M$. The presented sweep energies are given to fewer than six digits at the $M=1000,2000$ level. While successive sweeps at fixed $M$ typically converge smoothly (see Fig. 3), the rate of convergence slows when the remaining sweep error is comparable to the intrinsic error associated with truncation to $M$ states. This slowdown results from the simple self-consistent sweep procedure employed in our DMRG algorithm. Consequently, given our computing re-

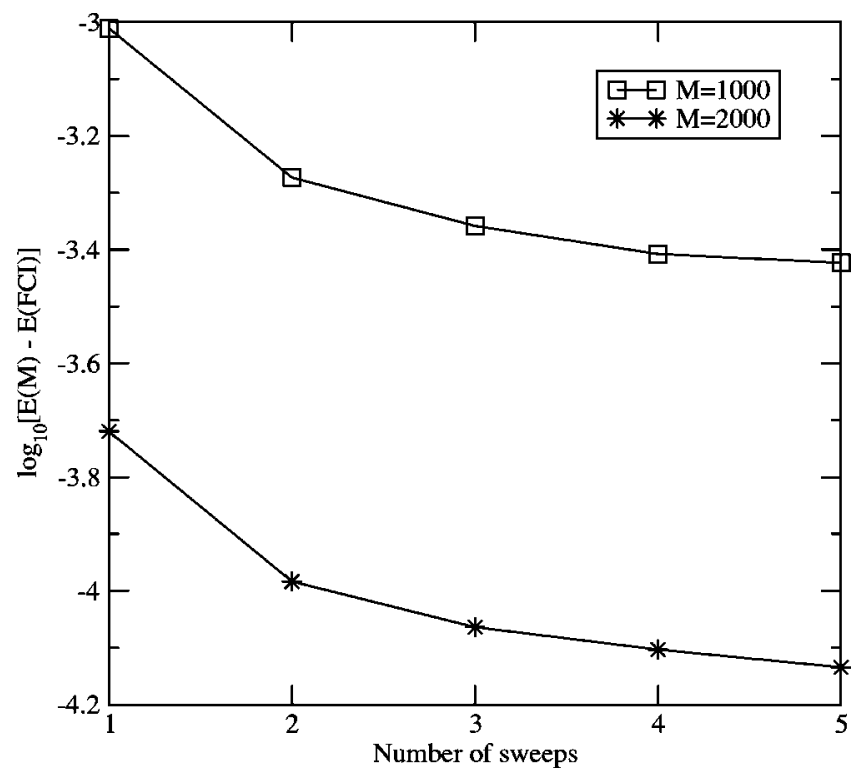

FIG. 2. Frozen-core energy errors for the nitrogen curve, measured relative to FCI energies. source constraints, it is wasteful to converge small $M$ calculations to very high accuracy. Convergence is also slower at stretched geometries, but we typically require no more than two to three times the number of sweeps to achieve the same level of convergence as at $r_{e}$. Acceleration schemes such as direct inversion in the iterative subspace (DIIS) ${ }^{34}$ may be used to improve the self-consistent DMRG sweep procedure in the future.

The DMRG energies at all $M$ values display an error across the potential energy curve that varies only within a factor of 2. The most accurate calculations at $M=4000 \mathrm{had}$ a maximum error of $20 \mu E_{h}$, and a nonparallelity error (the absolute difference between the maximum and minimum errors) of only $10 \mu E_{h}$. This is encouraging given the multireference nature of the problem, and demonstrates the insensitivity of the DMRG to the quality of the underlying RHF reference, which is very poor at stretched geometries.

The current results improve over our previous calculations in Ref. 12, which displayed an artificial increase in error in the intermediate bonding regime. This is due to our use of the improved sweep initialization procedure described in Ref. 14, which prevents the loss of quantum numbers during the early warm-up sweeps.

We now turn to our CC calculations. The earlier study of Krogh and Olsen ${ }^{1}$ already presented frozen-core FCI and CC calculations including up to quadruple excitations at a number of points along the nitrogen curve. We have, when possible, recomputed more fully converged FCI and CC energies, and extended the calculations of Krogh and Olsen to highly stretched geometries. We have been unable to obtain $\mathrm{CC}$ results using the RHF reference at the higher excitation levels at $4.2 a_{0}$. This is due, in part, to the poor convergence behavior of the $\mathrm{CC}$ equations. For example, at $4.2 a_{0}$, the CCSDT equations required over 500 iterations to converge, 


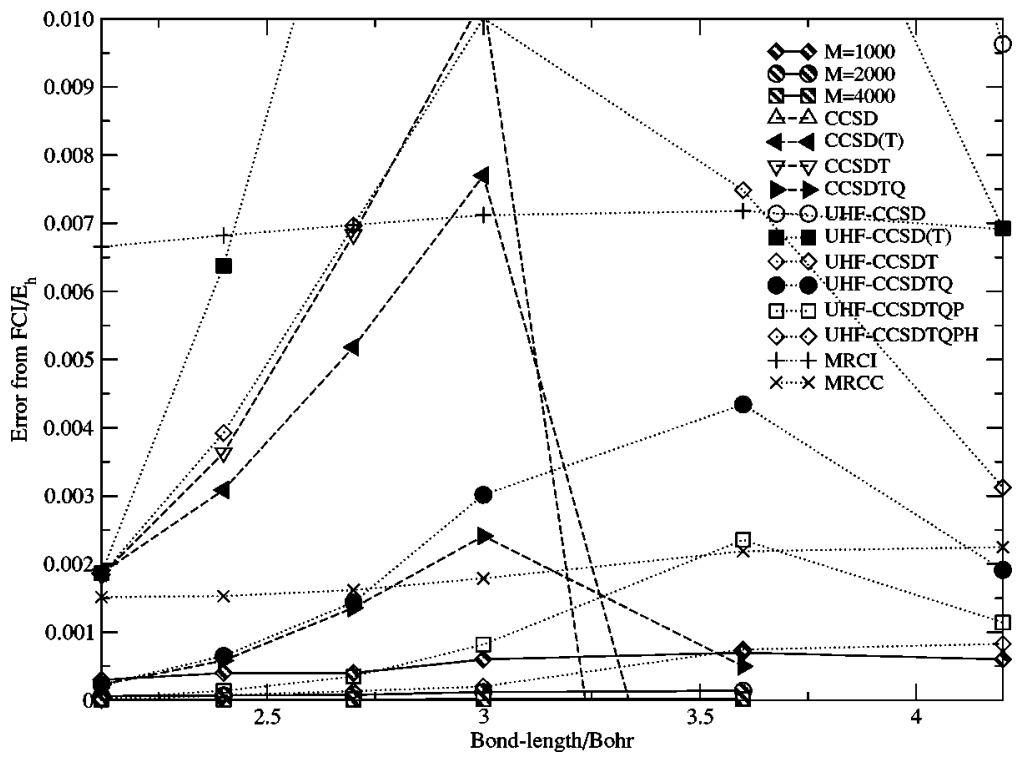

FIG. 3. Convergence of the DMRG energy/ $E_{h}$ (at fixed $M)$ as a function of the number of sweeps. and we could not converge the CCSDTQ energy even after many hundreds of iterations. This is a reflection of the breakdown of the restricted CC description at long bond lengths. In agreement with this breakdown, the error in the $\mathrm{CC}$ energies increases by an order of magnitude as the bond is stretched. Furthermore, the CCSDT energy actually lies significantly below the variational limit (Table II) at longer bond lengths. This pathological behavior contrasts with the behavior at equilibrium, where the convergence of the $\mathrm{CC}$ hierarchy is exponential and the CCSDTQPH energy is in error by only $2 \mu E_{h}$.

Although the unrestricted $\mathrm{CC}$ energies were obtained with an unrestricted frozen core, and are thus not strictly comparable with the other results, the effect of the change of core is quite small. To estimate this effect, we performed a frozen-core unrestricted FCI calculation at $2.4 a_{0}$, yielding a correlation energy (relative to the RHF reference) of $-0.371520 E_{h}, 66 \mu E_{h}$ above the restricted FCI result. This difference is small enough to allow us to comment on the change when going from the restricted to the unrestricted $\mathrm{CC}$ theory. In general, we observe a crossover between the accuracies of restricted $\mathrm{CC}$ and unrestricted $\mathrm{CC}$ energies at 3.0 $a_{0}$ (roughly $1.5 r_{e}$ ). However, although the unrestricted $\mathrm{CC}$ theories avoid a variational catastrophe at longer bond lengths, the convergence of the unrestricted $\mathrm{CC}$ hierarchy to the FCI result is still very slow. Thus, even with full hextuple excitations, the error at $4.2 a_{0}$ is $0.827 \mathrm{M} E_{h}, 400$ times larger than at $r_{e}$.

Finally, we comment briefly on our MRCI and MRCC results. Both theories produce well-behaved dissociation curves. It should be noted that although the absolute errors of the MRCI energies are far greater than the MRCC energies, the nonparallelity errors (NPEs) are within a factor of 2 . This is particularly significant given the greatly increased cost when going from MRCI to MRCC theory.

\section{All-electron calculations}

In Table III and Fig. 4 we present new all-electron calculations using the DMRG, CC, MRCI, and MRCC meth-

TABLE III. All-electron nitrogen correlation energies using DMRG and CC theories and UHF total energies. All results in hartrees. Core correlation energy $=\operatorname{DMRG}(M=4000)-\mathrm{FCI}($ frozen core $)$. Italics denote unconverged digits.

\begin{tabular}{|c|c|c|c|c|c|c|}
\hline & $2.118 a_{0}$ & $2.4 a_{0}$ & $2.7 a_{0}$ & $3.0 a_{0}$ & $3.6 a_{0}$ & $4.2 a_{0}$ \\
\hline UHF & -108.949378 & -108.891623 & -108.833687 & -108.790272 & -108.767549 & -108.775057 \\
\hline DMRG: 1000 & -0.3325 & -0.3498 & -0.3294 & -0.2985 & -0.2300 & -0.1946 \\
\hline DMRG: 2000 & -0.33271 & -0.35018 & -0.32978 & -0.29898 & -0.23039 & -0.19495 \\
\hline DMRG: 4000 & -0.332779 & -0.350263 & -0.329885 & -0.299103 & -0.230503 & -0.195033 \\
\hline CCSD & -0.318248 & -0.328171 & -0.297804 & -0.262607 & -0.208336 & -0.185187 \\
\hline $\operatorname{CCSD}(\mathrm{T})$ & -0.330927 & -0.343952 & -0.316958 & -0.278592 & -0.215287 & -0.187928 \\
\hline CCSDT & -0.330945 & -0.346407 & -0.323016 & -0.289165 & -0.222969 & -0.191795 \\
\hline CCSDTQ & -0.332565 & -0.349698 & -0.328577 & -0.296225 & -0.226187 & -0.193067 \\
\hline CCSDTQP & -0.332774 & -0.350209 & -0.329676 & -0.298450 & -0.228250 & -0.193901 \\
\hline CCSDTQPH-fc & -0.332794 & -0.350278 & -0.329890 & -0.299067 & -0.229859 & -0.194217 \\
\hline MRCISD & -0.325978 & -0.343302 & -0.322786 & -0.291872 & -0.223210 & -0.188013 \\
\hline MRCCSD & -0.331268 & -0.348739 & -0.328282 & -0.297336 & -0.228336 & -0.192808 \\
\hline Core & -0.00382 & -0.00349 & -0.00327 & -0.00317 & -0.00315 & -0.00314 \\
\hline
\end{tabular}




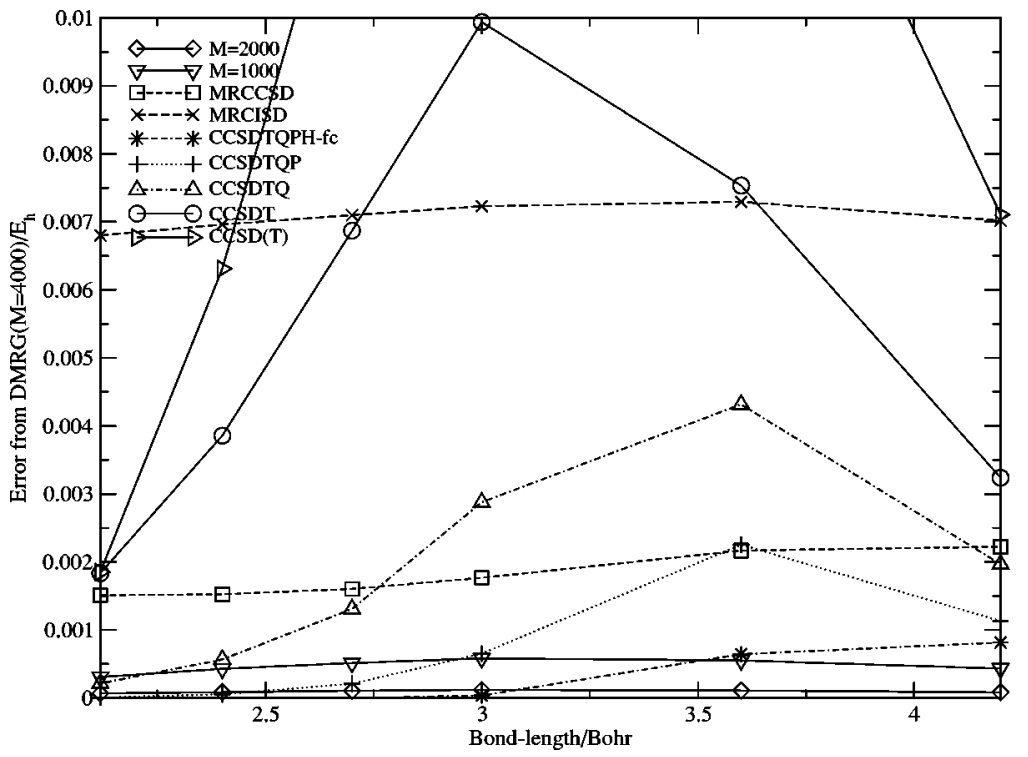

FIG. 4. All-electron energy errors for the nitrogen curve, measured relative to DMRG $(M=4000)$ energies. ods. We expect balanced results over the entire binding curve to be provided by our highest level DMRG $(M=4000)$ calculation. While we cannot give a definitive estimate of the error of our calculations in the absence of exact results, we can refer to our previous experience in the frozen-core calculations. At the frozen-core level at $r_{e}$, the DMRG $(M$ $=4000$ ) number is accurate to $9.5 \mu E_{h}$, while the CCSDTQPH energy is essentially exact. We observe a comparable energy difference of $15 \mu E_{h}$ between the all-electron DMRG $(M=4000)$ and CCSDTQPH- $f c$ numbers, indicating that errors in the correlation energies should be roughly transferable. From our frozen-core calculations, we also expect a slowly varying error in the DMRG energy across the potential energy curve; allowing the same factor of 2 variation in the error, we estimate that our DMRG $(M=4000)$ correlation energies are accurate to better than $30 \mu E_{h}$ across the nitrogen curve.

We note that we observe similar convergence (with $M$ ) of the DMRG algorithm both in the all-electron calculations, which used localized unrestricted orbitals, and the frozencore calculations which used delocalized restricted orbitals. Although a localized description might appear to be beneficial for the DMRG, in practice the advantages are reduced in systems which are not one dimensional, as is the case here.

The CC correlation energies lie below the DMRG ( $M$ $=4000$ ) energies at the geometries near equilibrium, with the crossover between the CCSDTQPH- $f c$ and DMRG $(M$ $=4000$ ) energies occurring at roughly $2.7 a_{0}$. However, as expected, the error in the single-reference $\mathrm{CC}$ calculations grows as the bond is stretched, which results from the poor quality of the Hartree-Fock reference at stretched geometries. Thus whereas the CCSDTQPH- $f c$ energy is expected to be accurate to a few $\mu E_{h}$ at $r_{e}$, at $4.2 a_{0}$ (roughly $2 r_{e}$ ), the energy lies about $0.84 \mathrm{M} E_{h}$ above the best DMRG result. Since the CCSDTQPH- $f c$ wave function contains the full active space, this NPE is attributable to the different treatment of dynamic correlation across the binding curve. Lower level CC methods display a characteristic maximum in the error near $3.6 a_{0}$. Furthermore, the commonly used CCSD
(T) approximation yields substantially different energies from CCSDT theory at stretched geometries.

The MRCISD and MRCCSD curves display large absolute errors (due to the incomplete treatment of dynamic correlation) but the error curves are quite flat, and the NPEs are relatively small in comparison to the error in the total energy. As the NPEs for both MR schemes are very similar, it appears that the size-consistency problem of MRCI is an unimportant issue for the calculation of the potential energy curve of $\mathrm{N} 2$.

Our all-electron calculations allow us to estimate the core-correlation energy. As expected, the core-correlation energy [estimated from the DMRG $(M=4000)$ energies] smoothly decreases as the bond is stretched, decreasing from $3.8 \mathrm{M} E_{h}\left(r_{e}\right)$ to $3.1 \mathrm{M} E_{h}\left(4.2 a_{0}\right)$.

Finally, a few remarks concerning the cost of these calculations. The time taken per DMRG sweep for the allelectron calculations was roughly $0.56 / 1.6 / 8.5 \mathrm{~h}$ at the $M$ $=1000 / 2000 / 4000$ levels using $641.05 \mathrm{GHz}$ Sun UltraSparc IIICu processors. It should be noted that the all-electron DMRG calculations are not much more expensive than the frozen-core calculations which take 0.391 .1 or $5.0 \mathrm{~h}$, respectively, at the same $M$ levels; the theoretical quartic scaling yields a cost ratio of 1.4 between all-electron and frozen core calculations. The CCSDTQPH- $f c$ calculations took roughly 1 day per $\mathrm{CC}$ iteration on a $2.4 \mathrm{GHz}$ Pentium IV workstation, with a total number of iterations ranging from 7 at $r_{e}$ to 100 at $4.2 a_{0}$.

\section{EXCITATIONS IN DMRG AND CC THEORY}

Although both the CC and DMRG hierarchies represent correlated wave functions in compact, and hence efficient forms, the two theories encompass significantly different models of correlations, with different strengths. As is well known, CC methods typically provide an efficient representation of correlated wave functions near the equilibrium region. While the CCSDTQPH- $f c$ wave function is still less compact than the DMRG $(M=4000)$ wave function, in that 


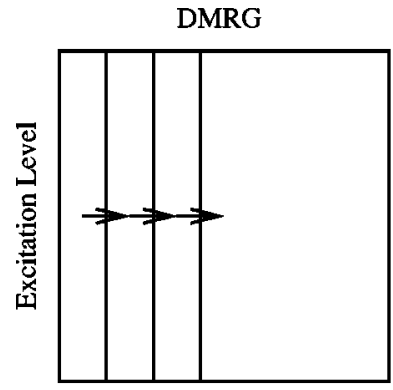

Orbital

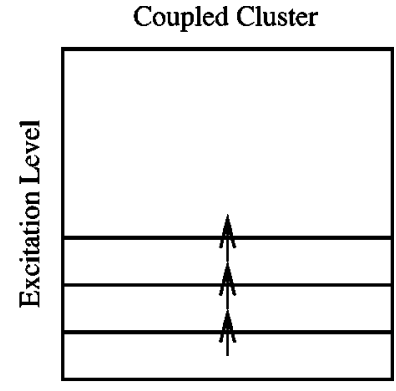

Orbital
FIG. 5. Excitations in DMRG and CC theory. In the DMRG, all levels of excitation to and from a given orbital are considered before truncation. In $\mathrm{CC}$ theory, excitations are truncated based on their order relative to the reference.

there are far more than $4000 \mathrm{CC}$ amplitudes [or even 300000 , the typical size of the full $O\left(16 M^{2}\right)$ tensor product DMRG space at the $M=4000$ level], we note that the high overhead associated with the RG methodology means that in practice it is typically more costly to perform a DMRG calculation of comparable accuracy to a high-level CC calculation at $r_{e}$. While the CC hierarchy makes use of a connected or disconnected representation of excitations, the DMRG makes no such distinction.

The weakness of the $\mathrm{CC}$ hierarchy lies in the favored status given to the reference system. Thus high-level excitations, as measured from the reference, are truncated first. This order of truncation is illustrated schematically in Fig. 5. In contrast, the DMRG provides a radically different scheme of truncation, where excitations are truncated to and from each given orbital, as illustrated in Fig. 5. Thus the DMRG treats all orbitals on an equal footing and is a true multireference theory. This accounts naturally for the much improved performance of the DMRG as compared to CC theory in multireference scenarios.

\section{CONCLUSIONS}

In this work we have carried out benchmark frozen-core and all-electron studies of the nitrogen molecule binding curve, using state-of-the-art DMRG and CC methods. Using the DMRG, we have been able to provide near-full-CIquality (within $30 \mu E_{h}$ ) solutions of the Schrödinger equation.

Our investigations demonstrate that the $\mathrm{CC}$ theories provide very good representations of correlation near equilibrium, but even with fully connected hextuple excitations, this description rapidly worsens at more stretched geometries. In contrast, the DMRG provides a much better balanced description across the full potential surface, although near the equilibrium geometry, this representation can be less efficient than that provided by $\mathrm{CC}$ theory.

\section{ACKNOWLEDGMENTS}

This work was supported by Christ's College, Cambridge, and the Fonds der Chemischen Industrie.

${ }^{1}$ J. W. Krogh and J. Olsen, Chem. Phys. Lett. 344, 578 (2001).

${ }^{2}$ W. D. Laidig, P. Saxe, and R. J. Bartlett, J. Chem. Phys. 86, 887 (1987).

${ }^{3}$ S. R. Gwaltney, E. F. C. Byrd, T. V. Voorhis, and M. Head-Gordon, Chem. Phys. Lett. 353, 359 (2002)

${ }^{4}$ X. Li and J. Paldus, Chem. Phys. Lett. 286, 145 (1998).

${ }^{5}$ S. A. Kucharski, J. D. Watts, and R. J. Bartlett, Chem. Phys. Lett. 302, 295 (1999).

${ }^{6}$ H. Larsen, J. Olsen, P. Jørgensen, and O. Christiansen, J. Chem. Phys. 113, 6677 (2000)

${ }^{7}$ T. H. Dunning, Jr., J. Chem. Phys. 53, 2823 (1989).

${ }^{8}$ S. R. White, Phys. Rev. Lett. 69, 2863 (1992).

${ }^{9}$ S. R. White, Phys. Rev. B 48, 10345 (1993).

${ }^{10}$ S. R. White and R. L. Martin, J. Chem. Phys. 110, 4127 (1999).

${ }^{11}$ A. O. Mitrushenkov, G. Fano, F. Ortolani, R. Linguerri, and P. Palmieri, J. Chem. Phys. 115, 6815 (2001).

${ }^{12}$ G. K.-L. Chan and M. Head-Gordon, J. Chem. Phys. 116, 4462 (2002).

${ }^{13}$ G. K.-L. Chan and M. Head-Gordon, J. Chem. Phys. 118, 8551 (2003).

${ }^{14}$ G. K.-L. Chan, J. Chem. Phys. 120, 3172 (2004).

${ }^{15}$ Ö. Legeza, J. Roder, and B. A. Hess, Mol. Phys. 101, 2019 (2003).

${ }^{16}$ The factor of 4 arises from the contribution of the Fock space of the orbital that is being added to the growing block.

${ }^{17}$ A. O. Mitrushenkov, R. Linguerri, P. Palmieri, and G. Fano, J. Chem. Phys. 119, 4148 (2003).

${ }^{18}$ Ö. Legeza and J. Sólyom, Phys. Rev. B 68, 195116 (2003).

${ }^{19}$ Antisymmetrization follows from the second-quantized operators used in the DMRG.

${ }^{20} \mathrm{~J}$. Gauss, in Encyclopedia of Computational Chemistry, edited by P. R. Schleyer, W. L. Jorgensen, H. F. Schaefer, III, P. R. Schreiner, and W. Thiel (Wiley, New York, 1998), p. 615.

${ }^{21}$ T. Crawford and H. Schaefer, Rev. Comp. Chem. 14, 33 (2000).

${ }^{22}$ M. Kállay and P. R. Surján, J. Chem. Phys. 113, 1359 (2000).

${ }^{23}$ M. Kállay and P. R. Surján, J. Chem. Phys. 115, 2945 (2001).

${ }^{24}$ S. Hirata and R. J. Bartlett, Chem. Phys. Lett. 321, 216 (2000).

${ }^{25}$ J. Olsen, J. Chem. Phys. 113, 7140 (2000).

${ }^{26}$ S. Hirata, J. Chem. Phys. 107, 9887 (2003)

${ }^{27}$ M. Kállay, P. G. Szalay, and P. R. Surján, J. Chem. Phys. 117, 980 (2002).

${ }^{28}$ N. Oliphant and L. Adamowicz, J. Chem. Phys. 96, 3739 (1992).

${ }^{29}$ J. Paldus and X. Li, Adv. Chem. Phys. 110, 1 (1999).

${ }^{30}$ R. D. Amos, A. Bernhardsson, A. Berning et al., MOLPRO, a package of $a b$ initio programs designed by H.-J. Werner and P. J. Knowles, release 2002.1 (2002)

${ }^{31}$ The small matrix sizes associated with higher symmetry groups led to memory fragmentation and loss of performance when running our code for extended periods. This is a feature of the dynamic memory allocation routine and not of our algorithm.

${ }^{32}$ J. Stanton, J. Gauss, J. Watts, W. J. Lauderdale, and R. J. Bartlett, Int. J. Quantum Chem. S26, 879 (1992).

${ }^{33}$ H. Lischka, R. Shepard, I. Shavitt et al., Columbus, an ab initio electronic structure program, release 5.9 (2001).

${ }^{34}$ P. Pulay, Chem. Phys. Lett. 73, 393 (1980). 